Pacific Journal of Mathematics

LINEAR MAPS OF THE DISK ALGEBRA 


\title{
LINEAR MAPS OF THE DISK ALGEBRA
}

\author{
RICHARD ROCHBERG
}

\begin{abstract}
Denote by $A$ the disk algebra, the sup-normed Banach algebra of functions continuous on the closed unit disk of the complex plane and analytic in the interior of the disk. This paper describes the elements of $K(F, G)$, the set of linear maps of $A$ into itself which are of norm one, fix the constants, and take the inner function $F$ to the inner function $G$. It is shown that any $L$ in $K(F, G)$ is determined by its action on a certain complement of $F A$ and that the image of this complement under $L$ must be orthogonal (in $H^{2}$ ) to $Z G A$. These facts are used to show that $K(F, G)$ is a compact convex set of real dimension at most $(n-1)(m+1)$ where $n$ and $m$ are the orders of $F$ and $G$ respectively. This result gives examples of non-multiplicative extreme points in the set of linear maps of $A$ into itself which are of norm one and fix the constants. Some analysis is made of $K(F, G)$ when $F$ and $G$ are not required to be inner.
\end{abstract}

Most of the maps considered will be of (operator) norm one and will fix the constants. We denote the set of all such maps by $K$. The most easily described elements of $K$ are those which are multiplicative; that is, those $T$ in $K$ which satisfy $T(f g)=(T f)(T g)$ for all $f$ and $g$ in $A$. The polynomials are dense in $A$, hence if $T$ is multiplicative then for all $f$ in $A$ and all $w$ in the closed disk, $(T f)(w)=$ $f(T(Z)(w))$ where $Z$ is the coordinate function on the disk. It is known that if $T$ is in $K$ and if $T(Z)$ is an extreme point of the unit ball of $A$, then $T$ is multiplicative [4] and is an extreme point of $K$ [3]. However, not all extreme points of the set $K$ are multiplicative. Lindenstrauss, Phelps, and Ryff, [2] have shown that the linear map of $A$ into itself defined by

$$
T f(\theta)=\frac{1}{2}\left[\left(1+\cos \frac{\theta}{2}\right) f\left(\frac{\theta}{2}\right)+\left(1-\cos \frac{\theta}{2}\right) f\left(\frac{\theta+2 \pi}{2}\right)\right]
$$

is non-multiplicative extreme point of $K$. It is immediate that $T$ is in $K$ and is not multiplicative. The not obvious fact that $T$ is an extreme point was verified directly. This map can alternatively be described by requiring that $T$ be an element of $K$ which satisfies the following conditions:

A. $T\left(Z^{2}\right)=Z$

B. $T\left(Z^{2} f\right)=Z T(f)$ for all $f$ in $A$, and

C. $T(Z)=1 / 2+(1 / 2) Z$.

This paper describes those elements of $K$ which satisfy conditions 
similar to $A$ above. It will be shown that if $T$ in $K$ satisfies $A$ then it satisfies $B$ and that there is a complex number $\alpha$ with $|\alpha| \leqq 1 / 2$ such that $T$ satisfies

$\mathrm{C}^{\prime} . \quad T(z)=\alpha+\bar{\alpha} Z$.

It will follow that an element of $K$ which satisfies $A$ is an extreme point of $K$ if and only if it satisfies $C^{\prime}$ with $\alpha$ a constant of modulus exactly $1 / 2$.

In general, condition $A$ will be modified to consider those elements of $K$ which take a non-constant inner function, $F$, to a non-constant inner function, $G$. We will denote the set of all such maps by $K(F, G)$. Thus $A$ will be replaced by

$\mathrm{A}^{\prime} . \quad T(F)=G$.

It is known [4] that if $T$ in $K$ satisfies $A^{\prime}$ then it also satisfies

B'. $T(F f)=G T(f)$ for all $f$ in $A$.

We will show that such a $T$ also satisfies a condition similar to, but a bit more lengthy than, $C^{\prime}$. Let the orders (number of zeros in the open unit disk) of $F$ and $G$ be $n$ and $m$ respectively. $K(F, G)$ will be shown to be isomorphic to a compact convex subset of a complex $n(m+1)$ dimensional vector space. It will also be shown that the coordinates of the points which correspond to $K(F, G)$ must satisfy certain additional restrictions and hence that $K(F, G)$ has real dimension at most $(n-1)(m+1)$. Finally, it will be shown that for certain specific choices of $F$ and $G$ the real dimension of $K(F, G)$ is exactly $(n-1)(m+1)$.

In $\$ 2$ the analogue of condition $C^{\prime}$ will be developed which will be used to show that an element of $K(F, G)$ is described by $n(m+1)$ complex parameters. Also, the relations will be developed which show that the element is actually determined by $(n-1)(m+1)$ real parameters. In $\S 3$ the structure of the set $K(F, G)$ will be described further for various specific choices of $F$ and $G$. In particular it will be shown that if $F=Z^{n}$ and $G=Z^{m}$, then the dimension $K(F, G)$ is given by the upper bound developed in $\S 2$. In $\S 4$ the results of $\S \S 2$ and 3 will be used to describe some of the extreme points of $K$. Section 5 indicates an alternative approach to describing linear maps that satisfy condition $A^{\prime}$. Although the method is more complicated than that of $\S 2$, it does not require $F$ or $G$ to be inner. Also, some of the complications which arise are of independent interest.

2. The elements $K(F, G)$. Let $F$ and $G$ be two non-constant inner functions in $A$ and let $T$ be an element in $K(F, G) . F, G$, and $T$ will be held fixed for the rest of this section. It will be assumed that the reader is familiar with the basic properties of $A$ and of the inner functions in $A$. (See [1] for these properties as well as for the basic facts about $H^{2}$ which will be used later.) Denote the zeros of 
$F$ by $\alpha_{1}, \cdots, \alpha_{n}$ and those of $G$ by $\beta_{1}, \cdots, \beta_{m}$. For conveniences we will proceed on the assumption that the $\alpha_{i}$ are distinct and that the $\beta_{j}$ are distinct. However, the results of this section, and, with minor modifications, the proofs remain valid for the case of multiplie zeros. For $i=1, \cdots, n$ let $A_{i}(z)=\left(z-\alpha_{i}\right) /\left(1-\bar{\alpha}_{i} z\right)$. For $j=1, \cdots, m$ let $B_{j}(z)=\left(z-\beta_{j}\right) /\left(1-\bar{\beta}_{j} z\right)$. Thus $F(z)=C \prod_{i=1}^{n} A_{i}(z)$ for some constant $C$ of modulus one and similarly $G(z)=D \prod_{j=1}^{m} B_{j}(z)$ where $D$ is a constant of modulus one. Without loss of generality we may assume that $C=1$ and be redefining $B_{1}(z)$ can write $G(z)=\prod_{j=1}^{m} B_{j}(z)$.

Denote the open unit disk of the complex plane by $D$, the closed disk by $\bar{D}$, and the unit circle by $\Gamma$. The functions in $A$ can be considered as functions on $\Gamma$ and we will sometimes write $f(\theta)$ for $f\left(e^{i \theta}\right)$.

Since $G$ is inner, it is an extreme point of the unit ball of $A$. Hence by a result in [4], $T(f F)=T(F) T(f)=G T(f)$ for all $f$ in $A$. Hence for any $f_{1}, f_{2}$ in $A$

$$
T\left(f_{1} \cdot\left(f_{2} \circ F\right)\right)=T\left(f_{1}\right) \cdot\left(f_{2} \circ G\right)
$$

where $h \circ k$ denotes the composite function, $(h \circ k)(z)=h(k(z))$. If $f_{2}$ is a polynomial this follows by induction on the degree of $f_{2}$. The general case follows from the density of the polynomials in $A$.

Let $e^{i \varphi}$ be a point of $\Gamma . \quad T$ is in $K$, hence the linear functional which sends $f$ to $(T f)\left(e^{i \varphi}\right)=(T f)(\varphi)$ is represented by a probability measure $\mu$ on $\Gamma$. Hence $T f(\varphi)=\int f(\theta) d \mu(\theta)$ for all $f$ in $A$. If we set $f=F$ we find $G(\varphi)=T F(\varphi)=\int F(\theta) d \mu(\theta)$. The left hand side is of modulus one and $F(\theta)$ is of modulus one on $\Gamma$ and $\mu$ is a probability measure. Hence the support of $\mu$ must be contained in $\{\theta: F(\theta)=$ $G(\varphi)$. Since $F$ maps $\Gamma$ onto itself in an $n$ to one manner, this is a finite set, $\left\{\theta_{1}, \cdots, \theta_{n}\right\}$. Thus there are positive constants $r\left(\theta_{1}\right), \cdots, r\left(\theta_{n}\right)$ such that

$$
T f(\varphi)=\sum_{F\left(\theta_{i}\right)=(i(\varphi)} r\left(\theta_{i}\right) f\left(\theta_{i}\right) .
$$

Let $S$ be a subset of $\{1, \cdots, n\}$. Let $H(z)=\prod_{i \in S} A_{i}(z)$. Note that $F / H$ is an element of $A$ and that on $\Gamma, F(z) / H(z)=F(z) \overline{H(z)}$. Hence, substituting in (2) and recalling that the $r\left(\theta_{i}\right)$ are real we have

$$
\begin{aligned}
T(F / H)(\varphi) & =\sum r\left(\theta_{i}\right) F\left(\theta_{i}\right) / H\left(\theta_{i}\right)=\sum r\left(\theta_{i}\right) F\left(\theta_{i}\right) \overline{H\left(\theta_{i}\right)} \\
& =G(\varphi) \sum r\left(\theta_{i}\right) \overline{H\left(\theta_{i}\right)}=G(\varphi) \overline{\sum r\left(\theta_{i}\right) H\left(\theta_{i}\right)} \\
& =G(\varphi) \overline{T H(\varphi)},
\end{aligned}
$$

or more conveniently,

$$
T(F / H)=T(F) \overline{T(H)} \text { on } \Gamma \text {. }
$$


Our goal now is to exploit equations (1) and (3). In order to do this it will be convenient to have bases for certain vector spaces at our disposal. For $j=1, \cdots, n$, set $C_{j}=\prod_{i=1}^{j} A_{i}$ Let $C_{0}=1$. We will need the following observation.

Proposition 1. The set of functions $C_{0}, \cdots, C_{n-1}$ is linearly independent. Furthermore, if $z_{1}, \cdots, z_{n}$ are any $n$ distinct points in $\bar{D}$, then the matrix $\left(C_{i-1}\left(z_{j}\right)\right)$ for $i, j=1, \cdots, n$ is nonsingular.

Proof. By comparing the location of zeros one sees that for $k=$ $0,1, \cdots, n-2, C_{k}$ is not in the span of $C_{k+1}, \cdots, C_{n-1}$, hence the linear independence. Next, suppose that the matrix $\left(C_{i \sim 1}\left(z_{j}\right)\right)$ is singular. Then there are constants $k_{1}, \cdots, k_{n}$, not all zero, so that the function $\sum_{i=1}^{n} k_{i} C_{i-1}(z)$ is zero for $z=z_{1}, \cdots, z_{n}$. Let $h(z)=\prod_{i=1}^{n-1}\left(1-\bar{\alpha}_{i} z\right)$. Then the function $L(z)=\sum k_{i} h(z) C_{i-1}(z)$ has zeros at $z=z_{1}, \cdots, z_{n}$. But $L(z)$ is a polynomial of degree at most $n-1$. Hence $L(z)$ is identically zero. Thus $\sum k_{i} C_{i-1}(z)$ is identically zero. Since the $C_{i}$ 's are linearly independent the $k_{i}$ 's must all be zero, contrary to assumption. This contradiction shows that the matrix is invertible and completes the proof.

It follows from this result that the functions $C_{i}, i=0, \cdots, n-1$ form a basis for the complement of $F A=\{f$ in $A: f=g F, g$ in $A\}$. For any $f$ in $A$ we can choose constants $a_{i}$ so that $f-\sum_{i=1}^{n-1} a_{i} C_{i}$ has zeros where $F$ does and hence is in $F A$. Hence the $C_{i}$ form the required basis. (A stronger result in this direction is presented in Theorem 1.) We will also need a basis for the complement of the space $Z G A=\{f$ in $A: f=Z G h, h$ in $A\}$. For our purposes it will be more convenient to look in the space $H^{2}$. Recall that $H^{2}$ is the Hilbert space of functions analytic in $D$ and such that

$$
\lim _{r \rightarrow 1} \int\left|f\left(r e^{i \theta}\right)\right|^{2} d \theta<\infty \text {. }
$$

For any $f$ in $H^{2}$ the radial $\operatorname{limit}_{\lim _{r \rightarrow 1}} f\left(r e^{i \theta}\right)=f(\theta)$ exists almost everywhere $(d \theta)$ and the inner product on $H^{2}$ can be given by $(f, g)=$ $1 / 2 \pi \int f(\theta) \overline{g(\theta)} d \theta$.

We know that $G=\prod_{i=1}^{m} B_{i}$. Define $D_{0}$ to be the constant function, $D_{0}=1$. For $k=1, \cdots, m$ let $D_{k}=\left(B_{k}-B_{k}(0)\right) \prod_{i=1}^{k-1} B_{i}$. Let $M$ be $(Z G A)^{\perp}$. That is, let $M$ be the orthogonal complement in $H^{2}$ of the subspace of $H^{2}$ generated by functions of the form $Z G f$ with $f$ in A.

Proposition 2. The functions $D_{i}, i=0, \cdots, m$ form an orthogonal basis for $M$. 
Proof. Since $A$ is an $H^{2}$ dense subspace of $H^{2}, M$ equals $\left(Z G H^{2}\right)^{\perp}$. This space is easily seen to be of dimension $m+1$, hence we need only show that the $D_{i}$ are pairwise orthogonal and that each $D_{i}$ is orthogonal to any function of the form $Z G Z^{n}$ for any nonnegative integer $n$. Both of these facts can be verified directly. We will verify the second. $\left(Z^{n+1} G, D_{i}\right)=1 / 2 \pi \int Z^{n+1} G \bar{D}_{i} d \theta$, but $Z^{n+1} G \bar{D}_{i}=f$, a function in $A$ which is zero at the origin. So

$$
\left(Z^{n+1} G, D_{i}\right)=\frac{1}{2 \pi} \int f(\theta) d \theta=f(0)=0 .
$$

Combining these observations gives the following:

Proposition 3. There are $(n-1)(m+1)$ complex constants $\left\{a_{i j}\right\}$ $i=1, \cdots, n-1 ; j=0, \cdots, m$ such that for $i=1, \cdots, n$

$$
T\left(C_{i}\right)=\sum_{j=0}^{m} a_{i j} D_{j}
$$

Proof. By the previous proposition it suffices to show that $T\left(C_{i}\right)$ is in $M=(Z G A)^{\perp}$. This, however, follows immediately from (3). We must show that for $n=0,1, \cdots\left(Z^{n+1} G, T\left(C_{i}\right)\right)=0$. By using (3)

$$
\begin{aligned}
\left(Z^{n+1} G, T\left(C_{i}\right)\right) & =\frac{1}{2 \pi} \int Z^{n+1} G \overline{T\left(C_{i}\right)} d \theta \\
& =\frac{1}{2 \pi} \int Z^{n+1} T\left(F / C_{i}\right) d \theta \\
& =\left(Z^{n+1} T\left(F / C_{i}\right)\right)(0) \\
& =0 .
\end{aligned}
$$

We have not yet shown that $T$ is uniquely determined by these constants nor have we ruled out the possibility that no choice of constants in (4) will give a map which is of norm one and hence in $K(F, G)$. The first of these issues is settled by the following decomposition formula.

TheOREM 1. Given $f$ in $A$ there are functions $f_{1}, \cdots, f_{n}$ in $A$ such that for all $z$ in $\bar{D}$

$$
f(z)=\sum_{i=0}^{n-1} C_{i}(z) f_{i}(F(z))
$$

Furthermore, the functions $f_{i}$ are uniquely determined by (5) and for each $i$ the mapping of $A$ into itself which sends $f$ to $f_{i}$ is continuous and linear. 
Proof. We will first show that we can find $f_{i}$ in $H^{2}$ so that (5) is satisfied for all $z$ in $D$. We will then show that we can conclude that these $f_{i}$ are actually in $A$.

Let $S$ be the linear operator on $H^{2}$ defined as multiplication by $F, S(f)=F f . \quad S$ is isometric and is not unitary on any non-trivial subspace of $H^{2}$. Hence, setting $J=\left(F H^{2}\right)^{\perp}$, the orthogonal complement of the range of $S, H^{2}=\sum_{k=0}^{\infty}\left(F^{k} J\right)$, the sum being a Hilbert space direct sum and the summands pairwise orthogonal. The space $J$ has dimension $n$. Let $v_{i}, i=1, \cdots, n$ be an orthonormal basis for $J$. Thus the elements $F^{k} v_{i}$ form an orthonormal basis for $H^{2}$. Pick $f$ in $A . f$ is also in $H^{2}$. Thus $f=\sum_{k, i} a_{k i} F^{k} v_{i}$. For each $i, i=1, \cdots, n$, define $f_{i}^{*}=\sum_{k=0}^{\infty} a_{k i} Z^{k}$. Since the sequence $a_{k i}$ is square-summable, the subsequence with the second index, $i$, fixed is square summable for each value of $i$. Thus the function $f_{i}^{*}$ is in $H^{2}$ for each $i$. The functions $f(z)$ and $\sum_{i=1}^{n} v_{i}(z) f_{i}^{*}(F(z))$ define the same element of $H^{2}$ since they have the same expansion with respect to the orthonormal basis consisting of the $F^{k} v_{i}$; i.e.

$$
f=\sum_{i} v_{i} \cdot\left(f_{i}^{*} \circ F\right) \text {. }
$$

Next we note that the $v_{i}$ can be written as linear combinations of the functions $C_{i}, i=0, \cdots, n$. To see this note that if $F(0) \neq 0$ then the functions $\left(C_{i}-1 \overline{\left.C_{i}(0)\right)}\right.$ for $i=1, \cdots, n$ form a basis for $J$. (If $F(0)=0$ then we can renumber the $A_{i}$ so that $A_{n}=Z$. In this case the functions $C_{i}, i=1, \cdots, n$ are themselves a basis for $J$.) Since the $v_{i}$ 's can be replaced by a linear combination of the $C_{i}$ 's, equation (6) can be rewritten in the form given by (5) with the $f_{i}$ 's in $H^{2}$. It is immediate from the construction that the functions $f_{i}$ are uniquely determined and depend linearly on $f$.

We will now show that the functions $f_{i}$ are actually continuous on $\bar{D}$. Fix $f$, pick $z_{0}$ in $\Gamma$. Pick $z_{k}, k=1, \cdots$ an arbitrary sequence of points in $D$ tending to $z_{0}$. Let $w(j, k) j=1, \cdots, n ; k=1, \cdots$ be the $F$ preimages of $z_{k}$ chosen so that $F(w(j, k))=z_{k}$ and $w(j, k)$ approaches $w(j, 0)$ as $k$ becomes infinite, where $w(j, 0), j=1, \cdots, n$, are the $n$ distinct $F$ preimages of $z_{0}$.

We know that $f$ is continuous on the closed disk. Hence, using (5) we have for $j=1, \cdots, n$

$$
\lim _{k \rightarrow \infty} \sum_{i} C_{i}(w(j, k)) f_{i}(F(w(j, k)))=f(w(j, 0)) .
$$

Hence, for the same values of $j$,

$$
\lim _{k} \sum_{i} C_{i}(w(j, k)) f_{i}\left(z_{k}\right)=f(w(j, 0)) .
$$

By deleting finitely many of the $z_{k}$ we can insure that for each 
$k$ the $n$ points $w(j, k)$ are distinct. Let $M(k)$ be the $n$ by $n$ matrix, $M(k)=\left(a_{i j}\right)=\left(C_{i}(w(j, k))\right)$. Since the $w(j, k)$ are distinct, Proposition 1 insures that $M(k)$ is invertible for each $k$. Let $V(k)$ be the vector $V(k)=\left(f_{1}\left(z_{k}\right), \cdots, f_{n}\left(z_{k}\right)\right)$. Let $W$ be the vector $(f(w(1,0)), \cdots, f(w(n, 0))$. The previous limit can now be rewritten

$$
\lim _{k \rightarrow \infty}(V(k) M(k))=W .
$$

Since the $C_{i}$ are continuous we know that $\lim M(k)=M(0)$ and that all of the $M(k)$ are invertible and that $\lim M(k)^{-1}=M(0)^{-1}$. Hence

$$
\begin{aligned}
W M(0)^{-1} & =\lim (V(k) M(k)) \lim M(k)^{-1} \\
& =\lim \left(V(k) M(k) M(k)^{-1}\right) \\
& =\lim (V(k)) .
\end{aligned}
$$

Thus $\lim V(k)$ exists and hence for each $i, \lim f_{i}\left(z_{k}\right)$ exists. Thus all of the $f_{i}$ are continuous at $z_{0}$. Since $z_{0}$ was arbitrary all of the $f_{i}$ are in $A$. Since every element of $A$ can be written in the form (5), it follows from the closed graph theorem that the $f_{i}$ depend continuously on $f$ and the theorem is proved.

For $F$ and $G$ as above, define $L(F, G)$ to be the set of all bounded linear maps of $A$ into itself which take $F$ to $G$. We will regard $C^{n(m+1)}$ as the space of $n$ by $m+1$ matrices, $\left(a_{i j}\right), i=0, \cdots, n-1$; $j=0, \cdots, m$. To each $N=\left(a_{i j}\right)$ in $C^{n(m+1)}$ we can associate $\varphi(N)$ in $L(F, G)$ defined by requiring that $\varphi(N)(1)=\sum_{j} a_{0 j} D_{j}$, that equation (4) be satisfied, and that if $f$ is rewritten as in equation (5), then

$$
\varphi(N)(f)=\sum_{i}\left(\varphi(N)\left(C_{i}\right)\right)\left(f_{i} \circ G\right) .
$$

If the first row in $N$ is $(1,0,0, \cdots, 0)$ then the description of $\varphi(N)$ simplifies $-\varphi(N)$ is the unique element of $L(F, G)$ which satisfies (1) and (4). (Note that equation (1) implies that $\varphi(N)(1)=1$.)

THEOREM 2. The mapping $\varphi$ is a well defined, one-to-one, linear, continuous mapping of $C^{n(m+1)}$ into $L(F, G)$. The set $K(F, G)$ is contained in the range of $\varphi$ and $\varphi^{-1}(K(F, G))$ is a compact convex subset of $C^{n(m+1)}$ of real dimension at most $(n-1)(m+1)$.

Proof. The basic properties of $\varphi$ follow from the definition and the previous theorem. The fact that $K(F, G)$ is contained in the range of $\varphi$ follows from Proposition 3. The preimage of $K(F, G)$ is clearly closed. To see that it is bounded we define $n(N)$ for $N$ in $C^{n(m+1)}$ by $n(N)=\|\varphi(N)\|$. Direct verification shows that $n(\cdot)$ is norm.

$$
\varphi^{-1}(K(F, G)) \subseteq\{M: n(M)=1\} \text {. }
$$


Thus $\varphi^{-1}(K(F, G))$ is a bounded set in the $n(\cdot)$ topology. However, in a finite dimensional vector space any two norms are equivalent. Hence $\varphi^{-1}(K(F, G))$ is bounded in the ordinary norm and is compact.

We now show that $\varphi^{-1}(K(F, G))$ has real dimension at most $(n-1)(m+1)$. Fix $\theta$ in $\Gamma$. Fix $N$ in $C^{n(m+1)}$. Let $C(\theta)$ be the column vector $\left(\varphi(N) C_{0}(\theta), \cdots, \varphi(N) C_{n-1}(\theta)\right)^{t}$. Let $D(\theta)$ be the column vector $\left(D_{0}(\theta), \cdots, D_{m}(\theta)\right)^{t}$. We know that $C(\theta)=N D(\theta)$. However, if we assume that $\varphi(N)$ is in $K(F, G)$ then we have another representation for $C(\theta)$ given by equation (2). Namely we know that for $i=$ $0, \cdots, n-1$

$$
T\left(C_{j}\right)(\theta)=\sum_{F(\theta)=G(\theta)} C_{i}\left(\theta_{k}\right) r\left(\theta_{k}\right)
$$

and that the $r\left(\theta_{k}\right)$ are positive real numbers. Let $R(\theta)$ be the vector $\left(r\left(\theta_{1}\right), \cdots, r\left(\theta_{n}\right)\right)^{t}$ and $M(\theta)$ the matrix $\left(C_{i}\left(\theta_{k}\right)\right) i=0, \cdots, n-1 ; k=$ $1, \cdots, n$. Thus $C(\theta)=M(\theta) R(\theta)$. Hence $N D(\theta)=M(\theta) R(\theta)$ for all $\theta$ in $\Gamma$. Pick $\varphi_{0}, \cdots, \varphi_{n}$ in $\Gamma$ so that the vectors $D\left(\varphi_{0}\right), \cdots, D\left(\varphi_{m}\right)$ are linearly independent. To see that such a choice can be made note that if the set of vectors $\{D(\theta): \theta \in \Gamma\}$ lie in an $m$ dimensional subspace then the $m+1$ functions $D_{0}, \cdots, D_{m}$ must be linearly dependent on $\Gamma$ and hence in $A$ thus contradicting Proposition 2. Hence

$$
N D\left(\varphi_{k}\right)=M\left(\varphi_{k}\right) R\left(\varphi_{k}\right)
$$

for $k=0, \cdots, m$. For fixed $k$, the left hand side of (7) ranges, a priori, over a space of complex dimension $m+1$ as $N$ varies over the subspace spanned by $\varphi^{-1}(K(F, G))$. However, for fixed $k$, since $R\left(\varphi_{k}\right)$ contains only real entries and these entries sum to one, the possible values for the right hand side of (7) are contained in a real affine space of dimension $n-1$. That is, there are $n-1$ column $n$-vectors, $e_{1 k}, \cdots, e_{n-1, k}$ so that $N D\left(\varphi_{k}\right)=(1,0, \cdots, 0)^{t}+\sum_{i} b_{i k} e_{i k}$ with the $b_{i k}$ real numbers. These $(n-1)(m+1)$ real numbers $b_{i k}, i=$ $1, \cdots, n-1 ; k=0, \cdots, m$ completely determine $N$; if $N$ and $N^{\prime}$ give rise to the same set of $b_{i k}$ then $N$ and $N^{\prime}$ agree on the $D\left(\varphi_{k}\right)$. The $D\left(\varphi_{k}\right)$ form a basis for the demain of $N$ and $N^{\prime}$, hence $N=N^{\prime}$. Thus the set of $N$ such that $\varphi(N)$ is in $K(F, G)$ lies in a real affine subspace of $C^{n(m+1)}$ of real dimension $(n-1)(m+1)$ and the proof is complete.

Two comments are in order. First, the previous theorem gives only an upper estimate for the dimension of $K(F, G)$. It does not eliminate the possibility that $K(F, G)$ may be empty, be a single point, or have real dimension strictly less than $(n-1)(m+1)$. To see why this is so, note that we have actually shown that in order for $\varphi(N)$ to be in $K(F, G), N$ must lie in an affine (real) subspace of $C^{n(m+1)}$ and must satisfy $n(N)=1$. We have not precluded the possibility 
that this subspace is exterior to the unit ball of the $n(\cdot)$ norm. Alternatively, we did not consider the requirement that the components of $R(\theta)$ must be positive.

Second, although we began by considering elements of $K(F, G)$ as linear maps of $A$ into itself we have seen that all of the elements of $K(F, G)$ can be regarded as linear maps of $H^{2}$ into $H^{2}$ which take elements of $A$ into elements of $A$ and which are bounded linear operators on $H^{2}$.

3. Special cases. In this section we will investigate the set $K(F, G)$ for a number of particular choices of $F$ and $G$. First we will consider several cases in which $K(F, G)$ is relatively simple. Then we will describe how $K(F, G)$ simplifies when $F$ is a power of an element of $A$. In particular we will show that if $F=Z^{n}$ and $G=$ $Z^{m}$ then the set $K(F, G)$ has real dimension exactly $(n-1)(m+1)$. Finally we will consider the case $n=2$ and will make some comments on the general case.

\section{A. The trivial cases.}

1. $F=1$. $K(F, G)$ must be empty unless $G=1$ since, by definition, every element of $K(F, G)$ satisfies $T(1)=1$. If $F=G=1$ then $K(F, G)=K$ and the previous results give no information.

2. $G=1$. Equation (2) must hold. Hence $T f(\theta)=\sum_{i} r\left(\theta_{i}\right) f\left(\theta_{i}\right)$ where the $\theta_{i}$ are the $n$ points at which $F(\theta)=1$. By evaluating (2) with $f(z)=\prod_{j=2}^{n}\left(z-e^{i \theta_{j}}\right)$ we find $T f(\theta)=r\left(\theta_{1}\right) f\left(\theta_{1}\right)$. Although the factor $r\left(\theta_{1}\right)$ may depend on $\theta$ it is always real and positive. Hence $T f / f\left(\theta_{1}\right)$ is a function in $A$ which is real on the boundary. Hence $T f$ is constant and $r\left(\theta_{1}\right)$ must be constant also. Similarly for the other $r\left(\theta_{j}\right)$. Thus $T f(\theta)=\sum_{j=1}^{n} a_{i} f\left(\theta_{i}\right)$ where the $a_{i}$ are any $n$ nonnegative real constants which sum to one. Any $T$ in $K(F, 1)$ is of this form. The dimension of $K(F, 1)$ is exactly $n-1$.

3. $F=Z$. The previous results give the estimate $\operatorname{dim} K(Z, G) \leqq$ 0 . Thus $K(Z, G)$ is empty or is a single point. The mapping $T$ defined by $(T f)(z)=f(G(z))$ is the single element of $K(Z, G)$.

4. $F=A_{1}$. If $F$ consists of a single Blaschke factor, $F(z)=$ $A_{1}(z)=(z-\alpha) /(1-\bar{\alpha} z)$ then $T$ is in $K\left(A_{1}, G\right)$ if and only if $T T_{A_{1}}$ is in $K(Z, G)$ where $T_{A_{1}}$ is the automorphism of $A$ defined by $\left(T_{A_{1}} f\right)(z)=$ $f\left(A_{1}(z)\right)$. Thus this case reduces to the previous one and $K\left(A_{1}, G\right)$ consists of a single point.

\section{B. Repeated factors.}

If $F$ is of the form $F=H^{k}$ for some non-constant inner function $H$, then the previous description simplifies somewhat. If the factors of $F$ are arranged in the appropriate order then the functions $H^{j}$, 
$j=1, \cdots, k-1$ are all among the functions $C_{i}$ in terms of which the elements of $K(F, G)$ are described. In this case equation (3) implies that on $\Gamma$, for $j=1, \cdots, k-1$,

$$
T\left(H^{k-j}\right)=T(F) \overline{T^{\top}\left(H^{3}\right)} .
$$

The previous results showed that the $T\left(C_{i}\right)$ cannot be chosen independently of each other. This equation puts the restrictions on the $T\left(C_{i}\right)$ in a particularly tractable form, especially if $F=Z^{n}$.

5. $F=Z^{n}, G=Z^{m}$. In this case equation (4) shows that $T$ in $K(F, G)$ must be determined by the constants $a_{i j}, i=1, \cdots, n-1$; $j=0, \cdots, m$ where

$$
T\left(Z^{i}\right)=\sum_{j} a_{i j} Z^{j}
$$

Equation (8) shows that on $\Gamma, T\left(Z^{n-i}\right)=Z^{m} \overline{T\left(Z^{i}\right)}=Z^{m} \sum_{j} \overline{a_{i j} Z^{j}}=$ $\sum \overline{a_{i j}} Z^{m-j}=\sum \bar{a}_{i(m-j)} Z^{j}$. But $T\left(Z^{n-i}\right)=\sum_{j} a_{(n-i) j} Z^{j}$. Thus for all $i$ and $j$ we must have $\bar{a}_{i, m-j}=a_{n-i, j}$ or equivalently,

$$
\overline{a_{i j}}=a_{n-i, m-j} \cdot
$$

We had previously found that the $(n-1)(m+1)$ complex numbers $a_{i j}$ must be restricted to a real subspace of real dimension $(n-1)(m+1)$. However an explicit description of the subspace was not given. In this case the subspace is exactly the set of vectors in complex $(n-1)(m+1)$ space which satisfy $(10)$. We will now show that the bounded subset of that subspace which corresponds to elements of $K(F, G)$ actually has full dimension.

Proposition 4. The set $K\left(Z^{n}, Z^{m}\right)$ has real dimension exactly $(n-1)(m+1)$.

Proof. We know that the dimension is at most this number. We now construct $(n-1)(m+1)$ independent elements. The construction is the direct generalization of that used in [2] in the case $n=2$, $m=1$. $n$ and $m$ are fixed. Pick $k$ and $l$ integers and subject to the following inequalities. If $n-1$ is even then $1 \leqq k \leqq(n-1) / 2$ and $0 \leqq l \leqq m$. If $n-1$ is odd then either $1 \leqq k<(n-1) / 2$ and $0 \leqq l \leqq m$, or else $k=n / 2$ and $0 \leqq l \leqq(m+2) / 2$. Thus we have $(n-1)(m+1) / 2$ or $((n-1)(m+1)+1) / 2$ pairs $(k, l)$ depending on whether $(n-1)(m+1)$ is even or odd. For $n, m, k, l$ fixed, for $\theta$ in $\Gamma$ and for $j=1, \cdots, n$ define $\theta_{j}=(m \theta+2 \pi(j-1)) / n$. For $f$ in $A$ define $T f$ by

$$
T f(\theta)=\sum_{j=1}^{n} \frac{1}{n}\left(1+\cos \left(k \theta_{j}-l \theta\right)\right) f\left(\theta_{j}\right) .
$$


Each of the coefficients of the $f\left(\theta_{j}\right)$ in (11) is positive. Hence to show that $T$ is in $K\left(Z^{n}, Z^{m}\right)$ it suffices to show that $T(1)=1, T\left(Z^{n}\right)=Z^{m}$, and that $T\left(Z^{s}\right)$ is in $A$ for $s=1, \cdots, n-1$. Pick $h$ with $0 \leqq h \leqq n$.

$$
\begin{aligned}
T\left(Z^{h}\right)(\theta) & =\sum \frac{1}{n}\left(1+\cos \left(k \theta_{j}-l \theta\right)\right)\left(\exp \left(i \theta_{j}\right)\right)^{h} \\
& =\sum\left(\frac{1}{n}+\frac{1}{2 n} \exp \left(i\left(k \theta_{j}-l \theta\right)\right)+\frac{1}{2 n} \exp \left(-i\left(k \theta_{j}-l \theta\right)\right)\right) \exp \left(i h \theta_{j}\right) \\
& =\frac{1}{n} \sum e^{i n \theta_{j}}+\frac{1}{2 n} e^{-i l \theta} \sum e^{i(h+k) \theta_{j}}+\frac{1}{2 n} e^{i l \theta} \sum e^{i(h-k) \theta_{j}} \\
& =\frac{1}{n} V_{h}(\theta)+\frac{1}{2 n} Z^{-l} V_{h+k}(\theta)+\frac{1}{2 n} Z^{l} V_{h-k}(\theta) .
\end{aligned}
$$

But $V_{s}(\theta)=n$ if $s=0, V_{s}(\theta)=0$ if $s=1, \cdots, n-1$, and $V_{s}(\theta)=n Z^{m}$ if $s=n$. Thus $T(1)=1, T\left(Z^{n}\right)=Z^{m}, T\left(Z^{n-k}\right)=(1 / 2) Z^{m-l}, T\left(Z^{k}\right)=$ $(1 / 2) Z^{l}$, and $T\left(Z^{s}\right)=0$ for all other values of $s$ (with the obvious modifications if $n=2 k)$. Thus $T$ is in $K\left(Z^{n}, Z^{m}\right)$ and if $T$ is expressed in the form (9) then $T$ corresponds to the matrix $\left(a_{i j}\right)$ with $a_{k l}=$ $a_{n-k, m-l}=1 / 2$ and all other entries zero. Similarly, if the "cosine" in equation (11) is replaced by "sine" the result is another element $T$ of $K\left(Z^{n}, Z^{m}\right)$ which corresponds to the matrix $\left(a_{i j}\right)$ with $a_{k l}=$ $(1 / 2) i, a_{n-k, m-l}=-(1 / 2) i$ and all other coefficients zero. Letting $k$ and $l$ range over the prescribed values and using the choices cosine and sine in (11), we obtain $(n-1)(m+1)$ elements of $K\left(Z^{n}, Z^{m}\right)$. By comparing the corresponding matrices it is clear that these elements are linearly independent over the reals. (When $(n-1)(m+1)$ is odd, the choice $k=n / 2, l=m / 2$, using the sine gives the zero matrix. Hence the apparent extra element does not throw the count off.)

6. $F=A_{1}^{n}, G=B_{1}^{m}$. As with Case 4,

$$
\left(T_{B_{1}}\right)^{-1} \circ K\left(A_{1}^{n}, B_{1}^{m}\right) \circ T_{A_{1}}=K\left(Z^{n}, Z^{m}\right) \text {. }
$$

Hence this case reduces to the previous one.

7. $F=Z^{2}, G=Z$. This is the example considered in the introduction and is special case of Case 5. The two maps constructed in Proposition 4 are $T_{1}$ and $T_{2}$ determined by $T_{1}(Z)=(Z+1) / 2$ and $T_{2}(Z)=(i Z-i) / 2$.

\section{Other Cases}

8. $F=A_{1} A_{2}, G=Z$. It is possible to reduce this case to Case 7 as follows. For $i=1,2, A_{i}(z)=\left(z-\alpha_{i}\right) /\left(1-\bar{\alpha}_{i} z\right)$. Choose $A_{3}(z)=$ $e^{i \theta}(z-b) /(1-\bar{b} z)$ so that $A_{3}(c)=\alpha_{1}$ and $A_{3}(-c)=\alpha_{2}$ for some real constant $c$. Hence $F \circ A_{3}$ is an inner function with zeros at $c$ and $-c$. Thus $F \circ A_{3}(z)=e^{i \theta^{\prime}}\left(z^{2}-c^{2}\right) /\left(1-c^{2} z^{2}\right)=A_{4}\left(z^{2}\right)$. Let $R$ be any element of $K\left(Z^{2}, Z\right)$. Let $T_{1}$ be the unique element of $K\left(Z, A_{3}\right)$. Let $T_{2}$ be 
the unique element of $K\left(A_{4}, Z\right)$. Let $T=T_{2} R T_{1} . \quad T(F)=T_{2} R T_{1}(F)=$ $T_{2} R\left(F \circ A_{3}\right)=\left(T_{2} R\right)\left(A_{4} \circ Z^{2}\right)=T_{2}\left(R\left(A_{4} \circ Z^{2}\right)\right)=T_{2}\left(A_{4}\right)=Z$. Thus $T$ is in $K(F, G)$. Furthermore, different choices for $R$ will give different maps $T$. Thus $\operatorname{dim} K(F, G)=\operatorname{dim} K\left(Z^{2}, Z\right)=2$.

9. Construction by composition. If $F$ and $G$ are given it is sometimes possible to obtain some elements of $K(F, G)$ by choosing some non-constant inner function $H$ and noting that if $T_{1}$ is in $K(F, H)$ and $T_{2}$ is in $K(H, G)$ then $T_{2} T_{1}$ is in $K(F, G)$. For example, if $F=$ $Z^{n}$ and $G$ is arbitrary, then by choosing $H=Z$ and using the results of Cases 3 and 5 we find that $K\left(Z^{n}, G\right)$ has real dimension at least $n-1$ for any $G$. Similarly, using the results of Cases 3 and 8 we see that if $F=A_{1} A_{2}$ and $G$ is arbitrary then $K(F, G)$ has real dimension at least two.

It should be noted that the techniques above do not seem to be sufficient to rule out the possibility that $K(F, G)$ might be empty in some nontrivial cases.

4. Extreme points. Recall that $K$ is the set of all linear maps of $A$ into itself which are of norm at most one and which fix the constants. The relation between the extreme points of $K$ and those of the various $K(F, G)$ is given by the following proposition.

Proposition 5. If $T$ is in $K(F, G)$ for some $F$ and $G$ then $T$ is an extreme point of $K$ if and only if it is an extreme point of $K(F, G)$.

Proof. The only if statement is trivial. We will show that if $T$ is in $K(F, G), T=1 / 2\left(T_{1}+T_{2}\right)$ with $T_{1}$ and $T_{2}$ in $K$ then $T_{1}$ and $T_{2}$ are in $K(F, G)$. To do this it suffices to show that $T_{1}(F)=T_{2}(F)=$ $G$. Recall that if $f$ is in $A$ and of norm one then $f$ is an extreme point of the unit ball of $A$ if and only if $\int \log \left(1-\left|f\left(e^{i \theta}\right)\right|\right) d \theta=-\infty$. Hence $G$ is certainly an extreme point of the unit ball of $A$. We know that $1 / 2\left(T_{1}(F)+T_{2}(F)\right)=G$ and each $T_{i}(F)$ is of norm at most one. Since $G$ is an extreme point we must have $T_{1}(F)=T_{2}(F)=G$. We note for later reference that the only properties of $F$ and $G$ which were used were that $F$ is of norm one and that $G$ is an extreme point of the unit ball of $A$.

The value of this result is that $K(F, G)$ is a finite dimensional compact convex set. Hence $K(F, G)$ has extreme points, at least $\operatorname{dim}(K(F, G))+1$ of them, and is the closed convex hull of its extreme points. Since we have shown a number of the $K(F, G)$ to be nonempty, a number of extreme points of $K$ can be obtained in this way. In general, the extreme points so obtained will not be multiplicative. 
We have noted that if $K(F, G)$ has real dimension $d$ then $K(F, G)$ has at least $d+1$ extreme points. There is no reason, however, why there should not be uncountably many. The problem of finding all of the extreme points of $K(F, G)$ is equivalent to the problem of describing $K(F, G)$ completely. It is not clear how to do this in general but we will do it for the particular case $F=Z^{2}, G=Z$. (Hence by the comments in part 8 of the previous section, also for $F=A_{1} A_{2}, G=Z$.) We have seen that every element $T$ of $K\left(Z^{2}, Z\right)$ is completely determined by a single complex number $\alpha$, where $T(Z)=$ $\alpha+\bar{\alpha} Z$.

Proposition 6. The set of $\alpha$ such that there is a $T$ in $K\left(Z^{2}, Z\right)$ with $T(Z)=\alpha+\bar{\alpha} Z$ is $\{\alpha:|\alpha| \leqq 1 / 2\}$. Those $\alpha$ with $|\alpha|=1 / 2$ correspond to the extreme points of $K\left(Z^{2}, Z\right)$.

Proof. We must show that the linear map described as follows has norm one. Given $f$ in $A, f$ can be written uniquely in the form $f(z)=f_{1}\left(z^{2}\right)+z f_{2}\left(z^{2}\right)$ with $f_{1}$ and $f_{2}$ in $A$.

$$
(T f)(w)=f_{1}(w)+T(Z)(w) f_{2}(w) .
$$

The condition $|\alpha| \leqq 1 / 2$ is necessary to insure that the norm of $T(Z)$, and hence of $T$, is at most one. We must show sufficiency. It suffices to show that for any $w$ in $\Gamma$, and any $f$ in $A$ that $|T f(w)| \leqq$ $\max \{|f(\sqrt{w})|,|f(-\sqrt{w})|\}$. That is

$$
\left|f_{1}(w)+T(Z)(w) f_{2}(w)\right| \leqq \max \left\{\left|f_{1}(w)+\sqrt{w} f_{2}(w)\right|,\left|f_{1}(w)-\sqrt{w} f_{2}(w)\right|\right\} .
$$

If $f_{2}(w)=0$ we are done. Hence dividing by $f_{2}(w)$, it suffices to show that for any complex number, $c$,

$$
|c+T(Z)(w)| \leqq \max \{|c+\sqrt{w}|,|c-\sqrt{w}|\} .
$$

This condition is guaranteed if we show that $T(Z)(w)$ is on the closed line segment connecting $\sqrt{w}$ and $-\sqrt{w}$. But $T(Z)(w)=a \sqrt{w}+$ $(1-a)(-\sqrt{w})$ with $a=\operatorname{Re}(\bar{\alpha} \sqrt{w})+1 / 2$.

5. Locally multiplicative maps. Let $T$ be a linear map of $A$ into itself. If there is a nonconstant element $F$ in $A$ such that $T(F) \neq 0$ and for all $f$ in $A T(f F)=T(f) T(F)$, then we will say that $T$ is locally multiplicative at $F$. We will denote the set of all maps which are locally multiplicative at $F$ and for which $T(F)=G$ by $M(F, G)$. It is immediate that if $T$ is in $M(F, G)$ then $T(1)=1$. Furthermore, since $T$ is continuous we must have $\|G\| \leqq\|F\|$. To see this we note that for any positive integer $n,\|G\|^{n}=\left\|G^{n}\right\|=$ $\left\|(T F)^{n}\right\|=\left\|T\left(F^{n}\right)\right\| \leqq\|T\|\left\|F^{n}\right\|=\|T\|\|F\|^{n}$. 
For $F$ in $A$ of norm one and $G$ in $A$, define $K(F, G)$ to be the set of all elements $T$ of $K$ for which $T(F)=G$. (If $F$ and $G$ are inner then this agrees with the previous definition.)

If $G$ is an extreme point of the unit ball of $A$ then the results of [4] guarantee that $K(F, G) \subseteq M(F, G)$ and the comment after Proposition 5 shows that that proposition is valid for $K(F, G)$

Fix $F$, a non-constant function in $A$ of unit norm, $G$ in $A$ and $T$ in $M(F, G)$. The basic fact we will use to analyze such $T$ is the observation that given any $w$ in $\bar{D}$ the linear functional, $l$, on $A$ which sends $f$ to $T f(w)$ contains in its kernel the ideal generated by $f-T f(w)$. One consequence follows immediately from this.

Proposition 7. If $G(\bar{D})$ is not contained in $F(\bar{D})$ then $M(F, G)$ is empty.

Proof. Suppose there is a point $w$ in $\bar{D}$ such that $G(w)$ is not in $F(\bar{D})$. Then $F-G(w)$ is bounded away from zero and hence is invertible in $A$. Thus the ideal generated by $F-G(w)$ is all of $A$. Hence, if $T$ is in $M(F, G)$ then $T f(w)=0$ for all $f$ in $A$. But $T 1(w)=$ 1. This contradiction shows that there is no such $T$.

The ideal generated by $F-T F(w)$ is especially tractable if $F-T F(w)$ has only finitely many simple zeros.

THEOREM 3. Given $T$ in $M(F, G)$ and $w_{0}$ in $D$ such that $F-G\left(w_{0}\right)$ has only finitely many zeros and all of these are simple zeros in $D$, then, denoting these zeros by $z_{1}, \cdots, z_{n}$, there are constants $k\left(z_{i}\right)$ such that for all $f$ in $A$

$$
T f\left(w_{0}\right)=\sum_{i=1}^{n} k\left(z_{i}\right) f\left(z_{i}\right) \text {. }
$$

Furthermore, if $G^{\prime}\left(w_{0}\right) \neq 0$ then for $w$ in some open neighborhood $N$ of $w_{0}$ there are analytic functions $z_{1}(G(w)), \cdots, z_{n}(G(w))$ such that $F\left(z_{i}(G(w))\right)=G(w), z_{i}\left(G\left(w_{0}\right)\right)=z_{i}$ and for all $f$ in $A$

$$
T f(w)=\sum_{i=1}^{n} k\left(z_{i}(G(w))\right) f\left(z_{i}(G(w))\right)
$$

where the $l_{i}\left(z_{i}(G(w))\right)$ are analytic with respect to $w$ in $N$.

Proof. Equation (12) is immediate. The hypotheses insure that there is a neighborhood $N$ of $G\left(w_{0}\right)$ such that $F^{-1}(N)$ consists of disjoint neighborhoods of the $z_{i}$ on each of which $F$ is analytic and univalent. The inverse functions of $F$, mapping $N$ to these neighborhoods, are the required functions $z_{i}(G(w))$. Equation (12) gives the required $k\left(z_{i}(G(w))\right)$. It remains to show that $k$ is analytic with 
respect to $w$. For $w$ in $N$, define $f_{w}(z)=\prod_{i=2}^{n}\left(z-z_{i}(G(w))\right) . \quad f_{w}$ depends analytically on $w$. Hence, by equation (13)

$$
T f_{w}(w)=k\left(z_{1}(G(w))\right) \prod_{i=2}^{n}\left(z_{1}(G(w))-z_{i}(G(w))\right) .
$$

Since $f_{w}$ depends analytically on $w$, so does $T f_{w}$ and hence so does the left hand side of the previous equation. The product on the right hand side of the previous equation is analytic in $w$ and is not zero at $w=w_{0}$. Hence $k\left(z_{1}(G(w))\right)$ is analytic with respect to $w$ near $w_{0}$. The argument is the same for the other indices and the theorem is proved.

Note that we have only shown that $k$ is analytic locally. Implicit in the above proof is the result that $k$ is analytic globally on some (possibly disconnected) Riemann surface lying over the disk.

If $G=Z$ then $k$ can actually be defined on a subset of the disk. However, even in this case the domain of definition of $k$ is rather complicated. The connected components of the domain of the $k$ produced in the previous theorem are contained in the connected components of $D-F^{-1}(F(\Gamma))$. If, however, further restrictions are placed on $F$, then $k$ can be shown to be meromorphic on $D-S$ where $S$ is a relatively small set. In particular suppose that there is a finite subset, $S_{1}$, of $\Gamma$ such that $F$ can be continued analytically past each point of $\Gamma-S_{1}$. Suppose further that for all $w$ in $\bar{D}, F^{-1}(w)$ is a finite set. Let $S=F^{-1}\left(F\left(S_{1}\right)\right)$.

THEOREM 4. If $F$ is as above and $T$ is in $M(F, Z)$ then there is a function $k$ such that

1. $k$ is meromorphic in $D-S$,

2. if $k$ has a pole at $z$ in $D-S$ then $F^{\prime}$ has a zero at $z$ and the order of the zero of $F^{\prime}$ at $z$ is greater than or equal to the order of the pole of $k$ at $z$,

3. if $F^{-1}(w)$ consists only of points of $D-S$ at which $F^{\prime} \neq 0$, then for all $f$ in $A$

$$
T f(w)=\sum_{F\left(z_{i}\right)=w} k\left(z_{i}\right) f\left(z_{i}\right),
$$

4. if $U$ is an open subarc of $\Gamma$ such that $F(U) \cong D-S$ and $F^{-1}(F(U))$ avoids $\left\{F^{\prime}=0\right\}$, then $k(z)=0$ for $z$ in $D-S$ near $U$.

Proof. Pick $z_{0}$ in $D-S$. If $F^{-1}\left(F\left(z_{0}\right)\right)$ consists of points of $D-S$ at which $F^{\prime}$ is nonzero then, by Theorem $3, k$ is defined and analytic in a neighborhood of $z_{0}$. Next, suppose that $z_{0}$ is in $D-S$ and $F^{\prime}\left(z_{0}\right) \neq 0$ and that the other preimages of $w_{0}=F\left(z_{0}\right)$, namely $z_{1}, \cdots, z_{n}$ are all in $\bar{D}-S_{1}$ and $F^{\prime}\left(z_{i}\right) \neq 0$. Let $\zeta_{0}, \cdots, \zeta_{n}$ be the 
$n+1$ inverses of $F$ at $w_{0}$ such that $\zeta_{i}\left(w_{0}\right)=z_{i}$. (Note that $\zeta_{i}$ is analytic even if $z_{i}$ is in $\Gamma-S_{1}$ ) $\quad$ For $w$ near $w_{0}$ set $f_{w}=\prod_{i=1}^{n}(z-$ $\left.\zeta_{i}(w)\right) . \quad k$ is already defined in a dense open subset of a neighborhood of $z_{0}$. At those points $z$, near $z_{0}$, at which $k(z)$ is already defined we have

$$
T f_{F(z)}(F(z))=k\left(\zeta_{0}(F(z))\right) \prod_{i=1}^{n}\left(\zeta_{0}(F(z))-\zeta_{i}(F(z))\right) .
$$

Hence, for $z$ near $z_{0}$, we can define

$$
k(z)=T f_{F(z)}(F(z)) / \prod_{i=1}^{n}\left(\zeta_{0}(F(z))-\zeta_{i}(F(z))\right) .
$$

This definition agrees with the previous definiton of $k$ where both are defined and shows $k$ to be analytic with respect to $z$.

We now have $k$ analytic in the required domain. If $z_{0}$ is a point of $D-S$ at which $k$ has not been defined then $F^{-1}(F(z))$ must contain some point at which $F^{\prime}=0$. For $w$ near $w_{0}=F\left(z_{0}\right)$ let $\zeta_{0}(w), \cdots, \zeta_{n}(w)$ he the $n+1 F$-preimages of $w$. Although the $\zeta_{i}$ cannot all be chosen to be analytic, they can be chosen so that $\zeta_{0}(w), \cdots, \zeta_{d}(w)$ all tend to $z_{0}$ as $w$ tends to $w_{0}$, where $d$ is the order of the zero of $F^{\prime}$ at $z_{0}$. Thus defining $f_{w}$ as before, we find that for $w$ near $w_{0}$, $M\|T\| \geqq\left\|T f_{w}\right\| \geqq\left|T f_{w}(w)\right|=\left|k\left(\zeta_{0}(w)\right) \prod_{i=1}^{n}\left(\zeta_{0}(w)-\zeta_{i}(w)\right)\right|$. Hence $k\left(\left(\zeta_{0}(w)\right)=0\left(\left|\zeta_{0}(w)-z_{0}\right|^{-d}\right)\right.$. Similarly, $\left|k\left(\zeta_{i}(w)\right)\right|=0\left(\left|\zeta_{i}(w)-z_{0}\right|^{-d}\right)$ for $i=0, \cdots, d$. Letting $w$ vary over a neighborhood of $w_{0}$ and $i$ range from 0 to $d, \zeta_{0}(w)$ varies over a neighborhood of $z_{0}$. Hence $k(z)=$ $0\left(\left|z-z_{0}\right|^{--d}\right)$ and $k$ has a pole of the required order. In particular, if $d=0$ then $k$ has a removable singularity at $z_{0}$.

We must now verify Part 4 of the theorem. Pick a point $z_{0}$ in the subarc $U$ given in the hypotheses. Let $w_{0}=F\left(z_{0}\right)$. Proceeding as before we construct a family of functions $f_{w}$ for $w$ near $w_{0}$ so that the $f_{w}$ are uniformly bounded, $f_{w}$ is zero on the $F$ preimages of $w$ not near $z_{0}$, the $f_{w}$ are bounded away from zero on the $F$ preimage of $w$ near $z_{0}$, and the $f_{w}$ depend analytically on $w_{0}$ Let $N$ be a neighborhood of $w_{0}$. T Tf $(w)$ is an analytic function of $w$ for $w$ in $N$. Let $\zeta(w)$ be the $F$ preimage of $w$ near $z_{0}$. For those $w$ with $\zeta(w)$ in $D$, a nonempty open subset of $N, T f_{w}(w)=k(\zeta(w)) f(\zeta(w))$. For those $w$ in $N$ for which $\zeta(w)$ is not in $D$, again a non-empty open subset of $N, T f_{w}(w)=0$. Thus $T f_{w}(w)$ must vanish identically in $N$. Hence $k(\zeta(w))$ is zero on $\zeta(N) \cap D$. Thus $k$ vanishes near $U$.

CoROLlary. If $F$ satisfies the hypotheses of the previous theorem and there is on subarc $U$ of $\Gamma$ such that $F(U) \subseteq D$ then $M(F, Z)$ is empty. 
Proof. Since $S$ is finite and the only cluster points of $F(\{z \in D$ : $\left.\left.F^{\prime}(z)=0\right\}\right)$ in $D$ must be in $S$, we can find $U^{\prime}$, a subarc of $U$ such that the hypotheses of Part 4 of the previous theorem hold for $U^{\prime}$. Hence, if there is a $T$ in $M(F, Z)$ then there is an associated $k$ meromorphic in a connected open subset of $D$. The previous theorem shows that $k$ vanishes identically on an open subset of its domain. Hence $k$ is identically zero. This is impossible, hence there is no such $T$.

COROLLARY. If $F$ satisfies the hypotheses of the previous corollary then $K(F, Z)$ is empty.

Proof. $Z$ is an extreme point of the unit ball of $A$, hence $K(F, Z)$ is contained in $M(F, Z)$ which, by the previous corollary, is empty.

This corollary is clearly not the best possible result of this type that can be obtained by these methods. For example, the restriction that $S$ be finite could be weakened. However it is not clear that similar methods would suffice to prove the following considerably more general result.

Conjecture. If $F$ in $A$ is of norm one and is not an inner function then $M(F, Z)$ is empty.

If $F$ is in fact inner and $\|T\|=1$ then the function $k$ of the previous theorem is relatively simple.

CoRollary. If $F$ is inner and $T$ is in $K(F, Z)$ then the function $k$ of Theorem 4 is rational.

Proof. $S$ is empty and hence $k$ is meromorphic in $D$. Equation (2) can be used to define $k$ as a real valued function on $\Gamma$. This extension is clearly a continuous extension. Thus $k$ meromorphic in $D$ and can be continuously extended to be real on $\Gamma$. Thus by the reflection principle, $k$ is meromorphic on the Riemann sphere and hence is rational.

For example, if $T$ is the element of $K\left(Z^{2}, Z\right)$ which sends $Z$ to $\alpha+\bar{\alpha} Z$ then an elementary calculation shows that $k(z)=\left(\alpha+\bar{\alpha} z^{2}\right) / 2 z$.

\section{REFERENCES}

1. K. Hoffman, Banach Spaces of Analytic Functions, Englewood Cliffs, N. J. 1962.

2. J. Lindenstrauss, R. R. Phelps and J. V. Ryff, Extreme Nonmultiplicative Operators, (Mimeograph 1968).

3. R. R. Phelps, Extreme positive operators and homomorphisms, Trans. Amer. Math. Soc., 108 (1963), 265-274. 
4. R. Rochberg, Which linear maps of the disk algebra are multiplicative, Pacific J. Math., 38 (1971), 207-212.

Received August 23, 1971.

WASHINGTON UNIVERSITY 


\section{PACIFIC JOURNAL OF MATHEMATICS}

\section{EDITORS}

H. SAMELSON

Stanford University

Stanford, California 94305

C. R. Hовву

University of Washington

Seattle, Washington 98105
J. DugundJI

Department of Mathematics University of Southern California

Los Angeles, California 90007

RICHARD ARENS

University of California

Los Angeles, California 90024

\section{ASSOCIATE EDITORS}

E. F. BECKENBACH

B. H. NeumanN

F. WOLF

K. YoSHIDA

\section{SUPPORTING INSTITUTIONS}

UNIVERSITY OF BRITISH COLUMBIA

CALIFORNIA INSTITUTE OF TECHNOLOGY

UNIVERSITY OF CALIFORNIA

MONTANA STATE UNIVERSITY

UNIVERSITY OF NEVADA

NEW MEXICO STATE UNIVERSITY

OREGON STATE UNIVERSITY

UNIVERSITY OF OREGON

OSAKA UNIVERSITY
UNIVERSITY OF SOUTHERN CALIFORNIA

STANFORD UNIVERSITY

UNIVERSITY OF TOKYO

UNIVERSITY OF UTAH

WASHINGTON STATE UNIVERSITY

UNIVERSITY OF WASHINGTON

AMERICAN MATHEMATICAL SOCIETY

NAVAL WEAPONS CENTER

Printed in Japan by International Academic Printing Co., Ltd., Tokyo, Japan 


\section{Pacific Journal of Mathematics}

\section{Vol. 44, No. $1 \quad$ May, 1973}

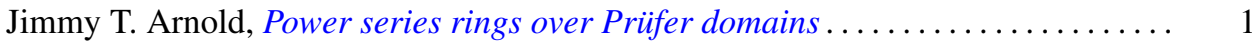

Maynard G. Arsove, On the behavior of Pincherle basis functions . . . . . . . . . 13

Jan William Auer, Fiber integration in smooth bundles ................. 33

George Bachman, Edward Beckenstein and Lawrence Narici, Function algebras

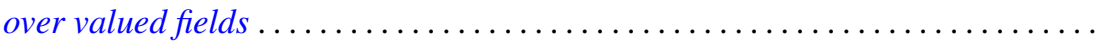

Gerald A. Beer, The index of convexity and the visibility function . . . . . . . . . . .

James Robert Boone, A note on mesocompact and sequentially mesocompact

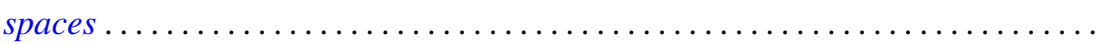

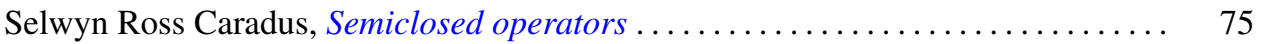

John H. E. Cohn, Two primary factor inequalities . . . . . . . . . . . . . . . 81

Mani Gagrat and Somashekhar Amrith Naimpally, Proximity approach to

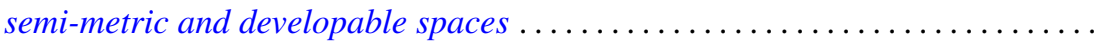

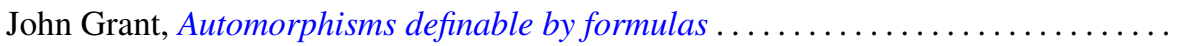

Walter Kurt Hayman, Differential inequalities and local valency ..............

Wolfgang H. Heil, Testing 3-manifolds for projective planes . . . . . . . . . . . . .

107

Melvin Hochster and Louis Jackson Ratliff, Jr., Five theorems on Macaulay

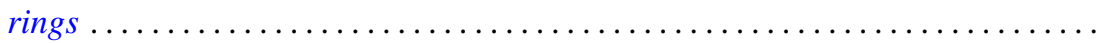

Thomas Benton Hoover, Operator algebras with reducing invariant subspaces ....

James Edgar Keesling, Topological groups whose underlying spaces are separable

Fréchet manifolds...

Frank Leroy Knowles, Idempotents in the boundary of a Lie group . .

191

George Edward Lang, The evaluation map and EHP sequences ...

201

Everette Lee May, Jr, Localizing the spectrum . . . . . . . . . . . .

211

Frank Belsley Miles, Existence of special $K$-sets in certain locally compact abelian groups.

Susan Montgomery, A generalization of a theorem of Jacobson. II . .

T. S. Motzkin and J. L. Walsh, Equilibrium of inverse-distance forces in

three-dimensions.

Arunava Mukherjea and Nicolas A. Tserpes, Invariant measures and the converse

of Haar's theorem on semitopological semigroups .

James Waring Noonan, On close-to-convex functions of order $\beta$

Donald Steven Passman, The Jacobian of a growth transformation

Dean Blackburn Priest, A mean Stieltjes type integral ........ .

Joe Bill Rhodes, Decomposition of semilattices with applications to topological

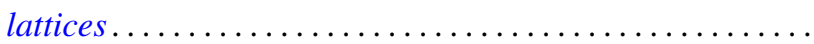

Claus M. Ringel, Socle conditions for $\mathrm{QF}-1$ rings ..........

Richard Rochberg, Linear maps of the disk algebra

Roy W. Ryden, Groups of arithmetic functions under Dirichlet convolution . .

Michael J. Sharpe, A class of operators on excessive functions

Erling Stormer, Automorphisms and equivalence in von Neumann algebras ..

Philip C. Tonne, Matrix representations for linear transformations on series

analytic in the unit disc. 